\title{
Spreading of a colon adenoma: a case report
}

\author{
Macaluso $\mathrm{D}^{1, \uparrow}$, Italia $\mathrm{F}^{2}$, Bianciardi $\mathrm{G}^{3 *, \uparrow}$ \\ ${ }^{1}$ Domenico Macaluso, Endoscopy Service, Ribera's Hospital, Agrigento, Italy \\ ${ }^{2}$ Fabrizio Italia, Laboratory of Pathology “OncoPath”, Augusta, Siracusa, Italy \\ ${ }^{3}$ Giorgio Bianciardi, Department of Medical Biotechnologies, Pathologic Anatomy, Siena University, Italy \\ tThese authors equally contributed to the study
}

\begin{abstract}
The capacity to spread beyond the limits of the tissue in which it was originated and to survive is the prerogative of malignant tumors, the feature at the basis of the concept of malignancy.

In $90 \%$ of cases, a colon cancer originates from its dysplastic precursor, the adenomatous polyp; evidence that $60-70 \%$ of that cancer is localized in the distal colon suggests that environmental factors affects the progression of the tumor, like the activation of HIF- 1 transcription factor and the physiological hypoxia that exists in this environment. Here we describe the finding of a clinical case where a benign tubulovillous adenoma appears able to survive and colonize the colonic mucosa away from the primary lesion. It was supported by visual inspection, histological evidence and fractal analysis (each tested morphometrical parameters- box dimension, information dimension, mass dimension, perimeter-area dimension, algorithmic complexity- overlapped each other, $\mathrm{p}<0.03$ ). If this finding will be confirmed in a high number of patients and by experimental studies, the current diagnostic/therapeutic approaches of the colon tumors should be modified, not only regarding colorectal adenomas, but also the superficial cancer of the large bowel.
\end{abstract}

\section{Introduction}

The mechanisms of spread of a malignant tumor in other tissues (metastasis) are well known since the previous century (bloodstream, lymphatic circulation, contiguity, via transcelomatic dissemination). It is a process affected by many factors, like as the ability to overcome hypoxia and to be provided with a vascular network [1-4].

During a colonscopy, two benign lesions (adenomas, one large vegetating proximal lesion and one small lesion, distally) were revealed in a patient, the position and the aspect at gross and microscopic levels suggesting that the small lesion could be derived from the other. In this work we face a clinical case that may suggest the possibility of an adenomatous polyp, a benign tumor, to spread and colonize the colonic mucosa remotely, with mechanisms considered, so far, prerogative of a malignant tumor.

\section{Case report}

During a colonoscopy performed at the Ribera's Hospital Endoscopy Service (Provincial Health Institution of Agrigento, Sicily) in a 63-year-old male that came to our attention for a positive Fecal Occult Blood Test (FOBT). Colonoscopy (Pentax EPM-3000 colonoscope) demostrated the presence of two eteroplastic lesions:

1) Near the cecum, the presence of a large vegetating lesion, dysplastic, lumpy but not ulcerated, with a short and thick peduncle; it invaded the intestinal lumen, and occupied the entire whole of it.

2) Approximately $20 \mathrm{~cm}$ distal to the above lesion, a small eteroplastic lesion located just below the hepatic flexure, lying on a colonic haustra. The mass, approximately $3 \mathrm{~mm}$ in diameter, was anchored to the colon mucosa through thin threadlike structures, and in relation with blood capillaries. The structure hadn't a vascular pole and at the detachment from the mucosa, the tumor appeared without a peduncle; capillaries, breaking away from the lumen of the colon, giving rise to a modest bleeding.

It was not possible to proceed with endoscopic resection due to the high dimension of the first lesion, and it was decided to perform some biopsies for histological/historphometric evaluations.

\section{Fractal analysis}

\section{Image analysis}

Hematoxylin-Eosin stained microscopic images were grabbed at $100 \mathrm{X}$ (Axioscop 40, Zeiss): five $0.25-\mathrm{mm}$ length mucosa samples randomly collected from three different sections for each lesion (corresponding to the presence of (about 1500 microstructures nuclei, mucin droplets, vacuoles - for each lesion) were digitized ( 1 pixel $=0.80$ micrometer). Aperture settings and conditions of illumination were kept constant. Images were $2 \mathrm{X}$ enlarged (Figure 1, top) and the contours were automatically extracted and converted to single pixel outlines by a canny-edge filter (Digital Image Magnifier software by Strikos Nikolaos: http://www.softoxi.com/digital-imagemagnifier.html), (fixed sigma, low and high threshold values, equals to $0.5,5$ and 10 , respectively) (Figure 1, bottom).

The obtained textures were characterized by analyzing their geometric complexity, entropy, mass dimension, perimeter-area dimension (Benoit 1.3 software, http://trusoft-international.com/ benoit.html) and algorithmic complexity.

Correspondence to: Giorgio Bianciardi, Department of Medical Biotechnologies, Pathologic Anatomy, Siena University, Italy, E-mail: giorgio.bianciardi@unisi.it

Received: October 25, 2015; Accepted: November 20, 2015; Published: November 25, 2015 

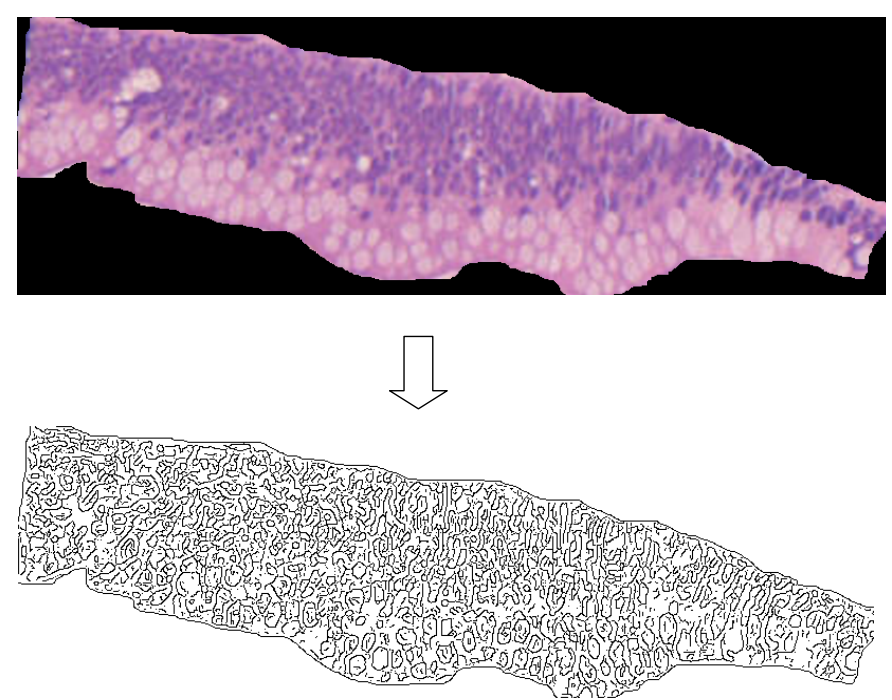

Figure 1. 0.25-mm length colonic mucosa sample (top) and its skeletonized contours obtained by a canny edge filter (bottom). About 300 microstructures are revealed (nuclei, mucin droplets, vacuoles).

\section{Geometric complexity (D0)}

Each image was covered by nets of square boxes (ranging from 5 to 100 pixels) and the amount of boxes containing any part of the outline was counted. A log-log graph was plotted of the side length of the square against the number of outline-containing squares. The log-log plot showed a line from 100 to 5 pixels with a high correlation coefficient, always above a value equal to 0.99 , thus justifying our fractal approach [5]. The slope of the linear segment of the graph represented the local fractal dimension (geometric complexity) of the image.

\section{Entropy (information dimension, D1)}

To evaluate the information (Entropy) present in the patterns, information dimension, D1, a robust estimate from a finite amount of data that gives the probability of finding a point in the image [6], was calculated. The set was covered with boxes of linear size, $d$ (from 5 to 100 pixels), keeping track of the mass, mi (the amount of pixels) in each box, and calculated the information entropy $I(d)$ from the summation of the number of points in the i-th box divided by the total number of points in the set multiplied for its logarithm. The slope of the log$\log$ plot of Information entropy vs. 1/box side length represented the information dimension of the distribution [5]. The log-log plot used to calculate the information dimensions showed a line from 100 to 5 pixels with a high correlation coefficient, always above a value equal to 0.99 , thus justifying our fractal approach.

\section{Mass dimension (Dm)}

To evaluate the mass dimension present in the pattern, a circle of radius $r$ was drawn on the skeletonized images, and the number of points in the set present inside circles of increasing radius were counted, M(r). The "mass" $\mathrm{m}(\mathrm{r})$ in the circle of radius $r$ was calculated as: $\mathrm{m}(\mathrm{r})=\mathrm{M}(\mathrm{r}) / \mathrm{M}$. The mass dimension, Dm, was the exponent in the following relationship: $\mathrm{m}(\mathrm{r}) \approx \mathrm{r}^{\mathrm{Dm}}$. The logarithm of $\mathrm{m}(\mathrm{r})$ versus the logarithm of $r$ was plotted. The slope represented the mass dimension of the distribution [5]. The log-log plot showed a line from 2 to 200 pixels with a high correlation coefficient, always above a value equal to 0.99 , thus justifying our fractal approach.

\section{Perimeter-area dimension (Dp)}

To estimate Dp, perimeter $\mathrm{P}$ and area $\mathrm{A}$ with boxes of different side length $\mathrm{d}$ were measured, and the logarithm of $\mathrm{A}$ on the vertical axis versus the logarithm of $\mathrm{P}$ on the horizontal axis was plotted and Dp was calculated from the slope of the straight line [5]. The loglog plot showed a line from 10 to 1000 pixels (perimeter), with a high correlation coefficient, always above a value equal to 0.99 , thus justifying our fractal approach.

\section{Algorithmic complexity (LZ)}

To determine the algorithmic complexity ("randomness") of the patterns, relative Lempel-Ziv, LZ, values were calculated according to the Kaspar and Schuster algorithm [7] using the Chaos Data Analyzer version 2.1 software package (CDA; Pro, Academic Software Library, North Carolina State University, USA). Briefly, patterns from a $251 \mathrm{x}$ 251 pixels window of the original image were transformed into 16732 points containing one dimensional vector, where each datum point was converted into a single binary digit according to whether the design is touched $(=1)$ or not $(=0)$, as described [8]. Relative L-Z values is close to 0 for a deterministic equation, close to 1 for totally destructured random phenomena.

The methodologies were always validated by measuring computer generated Euclidean and fractal shapes of known fractal dimensions.

\section{Statistical analysis}

Mean intra- and inter-observer coefficients varied $<3.0 \%$. Comparisons between groups were performed by variance analysis; regression analysis was applied in order to verify the linearity of the log-log plots.

\section{Results}

The small lesion and the large, vegetating lesion, showed fragments of a tubulovillous adenoma with low-grade epithelial dysplasia and small notes of dyskaryosis (nuclear atypia). The morphological pictures were substantially overlapping each other (Figure 2).

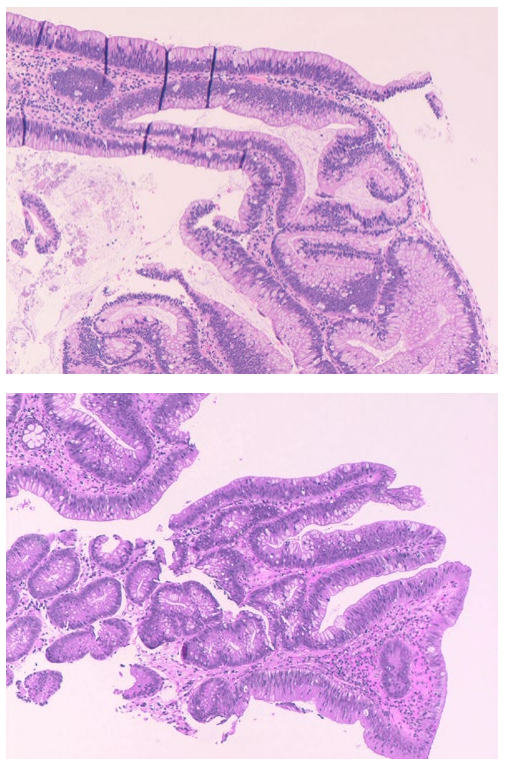

Figure 2. Large vegetative colonic proximal lesion taken at cecum level (top) and the distal small lesion presents below the hepatic flexure, lying on colic haustra (bottom). Both, present the aspect of a tubulovillous adenoma with low-grade epithelial dysplasia. Five fragments were randomly chosen from each lesion, $2 \mathrm{X}$ enlarged and submitted to image analysis (Figure 1). Hematoxilin-eosin, $100 \mathrm{X}$. 
The microscopic images of the lesions, small and large ones, presented a fractal structure, as demonstrated by the linear log-log plots (Figures 3 and 4).

In the two lesions, each tested morphometrical parameter (box dimension, information dimension, mass dimension, perimeter-area dimension, algorithmic complexity) overlapped each other (there were no significant differences between the groups, for each tested
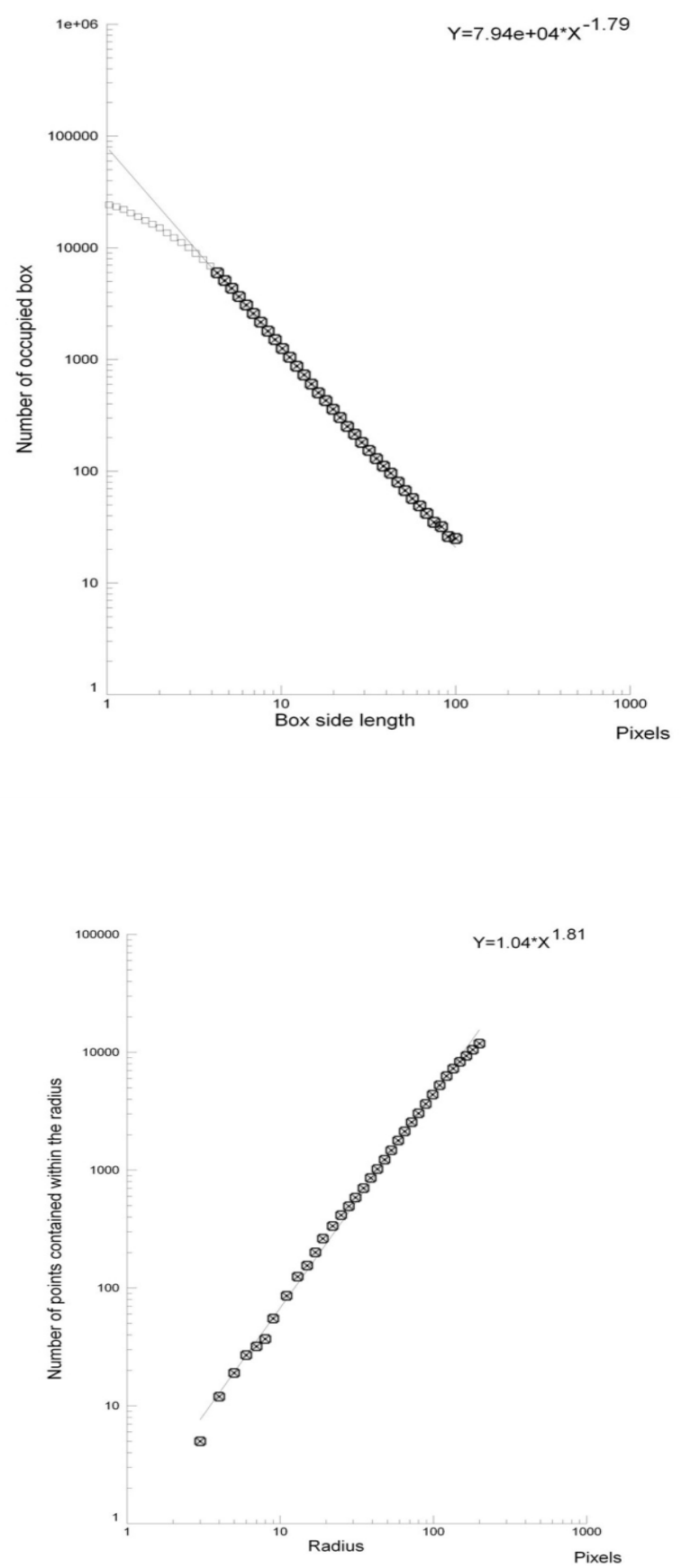

parameter: $p>0.05$, the probability of this occuring by chance is $1 / 2^{\wedge 5}$ $\mathrm{p}<0.03$, table 1$)$.

\section{Discussion}

In the present work, we describe a clinical case where a benign adenoma appears able to survive and colonize the colonic mucosa away from the primary lesion. This was supported by visual inspection
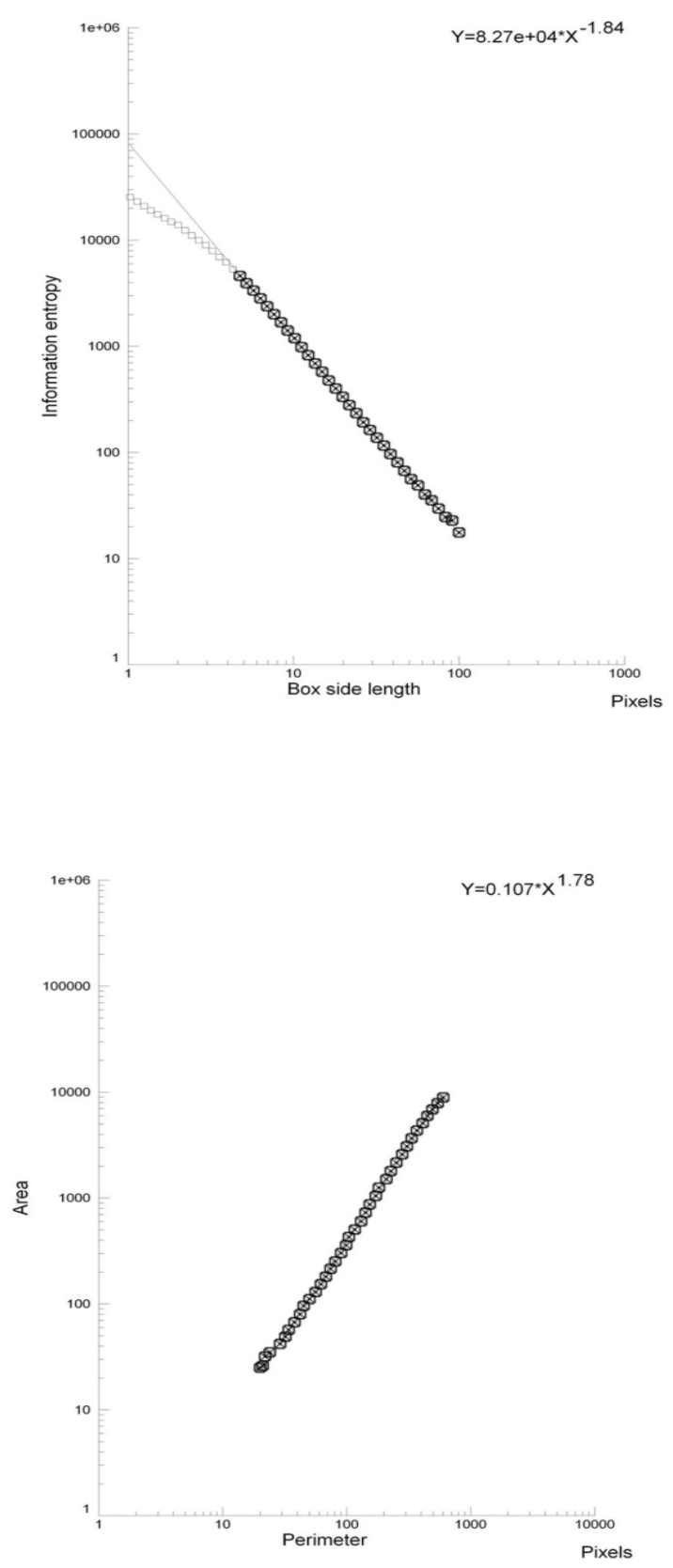

Figure 3. Log-log plots. Box-counting dimension-geometric complexity (top, left), information dimension-entropy (top, right), mass dimension (below, left), Perimeter/Area dimension (below, right). In the chosen fractal window a straight line is present: the analyzed structure (large adenoma at cecum level) is fractal. 

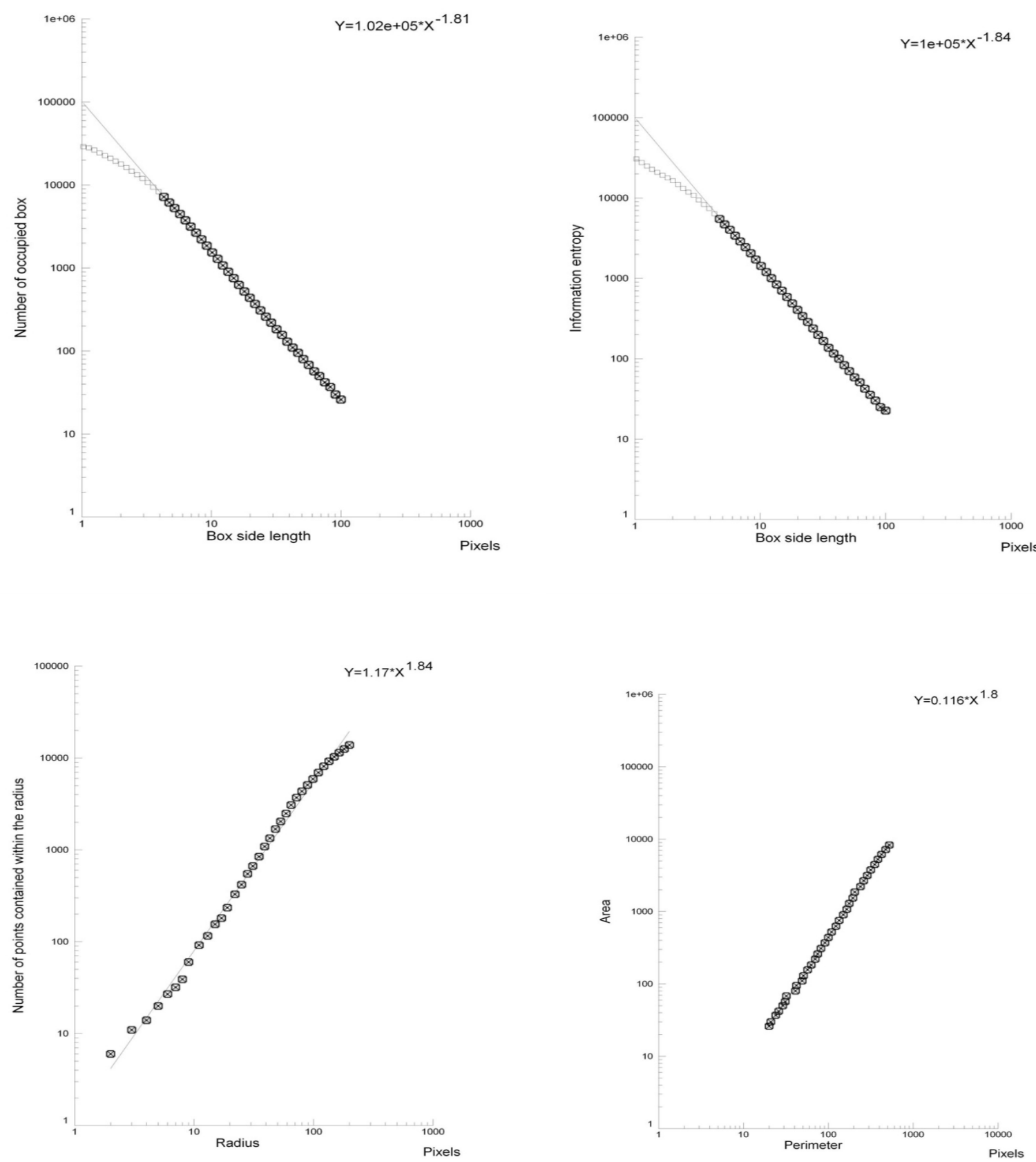

Figure 4. Log-log plots. Box-counting dimension- geometric complexity (top, left), information dimension - entropy (top, right), mass dimension (below, left), Perimeter/Area dimension (below, right). In the chosen fractal window a straight line is present: the analyzed structure (small adenoma, below the hepatic flexure) is fractal. Please, note the striking similarity of the fractal parameters (the slope of the straight line), large proximal lesion vs. small distal lesion (Figure $3 v s$. Figure 4).

Table 1. The large vegetating proximal lesion and the small distal lesion present overlapping fractal parameters (mean value/standard deviation), the probability of this occuring by chance is $\left.1 / 2^{\wedge}, \mathrm{p}<0.03\right)$.

\begin{tabular}{|l|c|c|}
\hline & $\begin{array}{c}\text { Large proximal } \\
\text { adenoma }\end{array}$ & Small distal adenoma \\
\hline Fractal Dimension (geometric complexity) & $1.80(0.02)$ & $1.77(0.04)$ \\
\hline Information Dimension (entropy) & $1.85(0.02)$ & $1.81(0.02)$ \\
\hline Mass Dimension & $1.84(0.07)$ & $1.79(0.11)$ \\
\hline Perimeter/Area Dimension & $1.75(0.06)$ & $1.74(0.06)$ \\
\hline Algorithmic complexity (Randomness) & $0.78(0.03)$ & $0.77(0.04)$ \\
\hline
\end{tabular}

during colonoscopy (presence of a small mass, distally to a large vegetating lesion; it was without a peduncle, vascular axis and vascular pole, anchored to the colon mucosa through thin threadlike structures, and in relation with nest of capillaries), histological evidence (the same microstructure of a tubulovillous adenoma with low-grade epithelial dysplasia and notes of dyskaryosis) and fractal analysis (five fractal parameters, obtained by analyzing about 1500 microstructures for each lesion, perfectly overlapped between the lesions). The small distal adenoma appears to be the daughter of the large vegetating proximal lesion. 
The Warburg effect, known since 1956, is the ability of cancer cells to respond to hypoxia, with an adaptation of their energy cycle. The colon mucosa is an anoxic environment, where a lot of different microaerophilic bacteria and archaebacteria live [9-11]. This could be a crucial step in the survival of that colonic neoplastic embolus, survival and adaptation being permitted by that anoxic environment. A key role should be played by the mitochondrial protein TRAP-1, with its ability to intervene on the transcription factor HIF-1 levels $[12,13]$. In normoxic conditions, this mediator is degraded by hydroxylation, whereas in neoplastic tissues TRAP-1 stabilizes HIF-1 [14]. In effect, immune histochemical investigations have shown that in malignant tumors there are significant high levels of HIF-1 in comparison to healthy tissues and if a metastasic process is present, still higher HIF-1 levels are present [15]. In this context, a kind of natural selection will favor the growth of the cellular clone adapted to the hypoxic stress [4]. We can recall that HIF-1 is a potent stimulator mitogen, specific for endothelial cells [16]; it produces changes of cellular metabolism, triggering the activation of glycolytic enzymes; leading angiogenesis and neovascularization, through vascular endothelial growth factor activation [15]. Significant should be also the role of nitric oxide, involved in the tone regulation of the newly formed vessels [17].

If our finding will be confirmed in a high number of patients, the current orientation to treat sessile adenomas of the colon by means of endoscopic sub-mucosal dissection $[18,19]$ must be revised. There is no doubt that early diagnosis of adenomatous polyps and their removal by endoscopic resection reduces the incidence of the colon carcinoma [20-23] but we must prevent the fragmentation of the polyp. Otherwise, the resection of the tumor could produce the primum movens of an iatrogenic spread of a colonic adenoma, due to the particular morphological characteristics of the colonic mucosa (folds, diverticula) and the presence of an anoxic environment. Even the slow intestinal transit, an increasing condition for the actual lifestyle, could help the engraftment of such adenomatous embolus. A dangerous situation for the patient, being the adenoma a benign lesion that can evolve in a malignant lesion [24]. Moreover, it may be also true for a superficial colon cancer, if treated by resection or endoscopic dissection: the residual fragments could be able to colonize contiguous or distal areas resulting in repetitive carcinomas.

Experimental studies might confirm our hypothesis. One should be carried out on cre-recombinase genetically modified mice, characterized by a higher incidence of tumors in the distal colon [25]. The experimental procedure should be obtained by taking tumor fragments from a proximal area of the colon and implant them by contact in the most distal areas, and then analyze the survival, engraftment and development of the cell aggregates. Another experimental procedure could be performed dosing HIF-1 levels in humans, checking its values in comparison to the $\mathrm{pO}_{2}$ levels [26] and dysplasia degree [22] in different parts of the colon.

\section{References}

1. Albenberg L, Esipova TV, Judge CP, Bittinger K, Chen J, et al. (2014) Correlation between intraluminal oxygen gradient and radial partitioning of intestinal microbiota. Gastroenterology 147: 1055-63. [Crossref]

2. Armougom F, Henry M, Vialettes B, Raccah D, Raoult D (2009) Monitoring bacterial community of human gut microbiota reveals an increase in Lactobacillus in obese patients and Methanogens in anorexic patients. PLoS One 4: e7125. [Crossref]

3. Höckel M, Vaupel P (2001) Tumor hypoxia: definitions and current clinical, biologic, and molecular aspects. J Natl Cancer Inst 93: 266-276. [Crossref]

4. Vaupel P (2004) The role of hypoxia-induced factors in tumor progression. Oncologist 9: 10-17. [Crossref]
5. Falconer K (1990) Fractal geometry: Mathematical Foundations and Applications. Chichester, John Wiley.

6. Pitsianis N, Bleris GL, Argyrakis P (1989) Information dimension in fractal structures. Phys Rev B 39: 7097-7100.

7. Kaspar F, Schuster HG (1987) Easily calculable measure for the complexity of spatiotemporal patterns. Phys Rev A 36: 842-848. [Crossref]

8. Bianciardi G, Traversi C, Cattaneo R, De Felice C, Monaco A, et al. (2012) Phase transition of the microvascular network architecture in human pathologies. Theor Biol Forum 105: 37-45. [Crossref]

9. Gaci N, Borrel G, Tottey W, O'Toole PW, Brugère JF (2014) Archaea and the human gut: new beginning of an old story. World J Gastroenterol 20: 16062-16078. [Crossref]

10. Gasbarrini A (2013) Il Microbiota Intestinale, Verducci ed.

11. Weaver GA, Krause JA, Miller TL, Wolin MJ (1986) Incidence of methanogenic bacteria in a sigmoidoscopy population: an association of methanogenic bacteria and diverticulosis. Gut 27: 698-704. [Crossref]

12. Fujita N, Markova D, Anderson DG, Chiba K, Toyama Y, et al. (2012) Expression of prolyl hydroxylases (PHDs) is selectively controlled by HIF-1 and HIF-2 proteins in nucleus pulposus cells of the intervertebral disc: distinct roles of PHD2 and PHD3 proteins in controlling HIF-1 $\alpha$ activity in hypoxia. J Biol Chem 287: 16975-16986. [Crossref]

13. Ke Q, Costa M (2006) Hypoxia-inducible factor-1 (HIF-1). Mol Pharmacol 70: 1469 1480. [Crossref]

14. Sciacovelli M, Guzzo G, Morello V, Frezza C, Zheng L, et al. (2013) The Mitochondrial Chaperone TRAP1 Promotes Neoplastic Growth by Inhibiting Succinate Dehydrogenase. Cell Metab 17: 988-999. [Crossref]

15. Zhong H, De Marzo AM, Laughner E, Lim M, Hilton DA, et al. (1999) Overexpression of Hypoxia-inducible Factor 1a in Common Human Cancers and Their Metastases. Cancer Res 59: 5830-5835. [Crossref]

16. Conway EM, Collen D, Carmeliet P (2001) Molecular mechanisms of blood vessel growth. Cardiovasc Res 49: 507-521. [Crossref]

17. Melillo G, Musso T, Sica A, Taylor LS, Cox GW, et al. (1995) A hypoxia-responsive element mediates a novel pathway of activation of the inducible nitric oxide synthase promoter. J Exp Med 182: 1683-1693. [Crossref]

18. Kudo S (1993) Endoscopic mucosal resection of flat and depressed types of early colorectal cancer. Endoscopy 25: 455-461. [Crossref]

19. Yamamoto H (2010) Endoscopic Submucosal Dissection for Colorectal Tumor. Gastrointest Res Basel Karger 27: 287-295.

20. Bertelson NL, Kalkbrenner KA, Merchea A, Dozois EJ, Landmann RG, et al. (2012) Colectomy for endoscopically unresectable polyps: how often is it cancer? Dis Colon Rectum 55: 1111-1116. [Crossref]

21. Diamond SJ, Enestvedt BK, Jiang Z, Holub JL, Gupta M, et al. (2011) Adenoma detection rate increases with each decade of life after 50 years of age. Gastrointest Endosc 74: 135-140. [Crossref]

22. Giacomin A (2010) Tumori del colon retto, Corso di base IRTUM, Tarquinia.

23. Uraoka T, Parra-Blanco A, Yahagi N (2013) Colorectal endoscopic submucosal dissection: is it suitable in western countries? J Gastroenterol Hepatol 28: 406-414 [Crossref]

24. Zamboni G, Lanza G, Risio M (1999) [Colorectal adenoma-carcinoma. Guidelines and minimal diagnostic criteria. Italian Group for Pathology of the Digestive System]. Pathologica 91: 286-294. [Crossref]

25. Xue Y, Johnson R, Desmet M, Snyder PW, Fleet JC (2010) Generation of a transgenic mouse for colorectal cancer research with intestinal cre expression limited to the large intestine. Mol Cancer Res 8: 1095-1104. [Crossref]

26. Zheng L, Kelly CJ, Colgan SP (2015) Physiologic Hypoxia and Oxygen Homeostasis in the Healthy Intestine. Am J Physiol Cell Physiol 309: C350-60. [Crossref]

Copyright: (C)2015 Macaluso D, Bianciardi G. This is an open-access article distributed under the terms of the Creative Commons Attribution License, which permits unrestricted use, distribution, and reproduction in any medium, provided the original author and source are credited. 